\title{
Determination of Optical Purity by Mass Spectrometry
}

\author{
Dong-Ung Lee, Klaus K. Mayer, and Wolfgang Wiegrebe ${ }^{* * *}$ ) \\ Faculty of Chemistry and Pharmacy, University, P. O. Box 397, \\ D-8400 Regensburg, Germany
}

Received April 14, 1987

MS-Isotope Dilution Analysis (MS-IDA) with deuterated 2 as standard was used to determine the optical purity of crystalline (+)-2, obtained from optically pure (+)-1 with ethyl chloroformate.

\section{Bestimmung der optischen Reinheit durch Massenspektrometrie}

Durch MS-Isotopenverdünnungsanalyse (MS-IDA) wurde die optische Reinheit von kristallinem (+)-2 bestimmt, das aus optisch reinem (+)-1 durch Chlorameisensäureethylester entsteht. Deuteriertes 2 diente als Standard.
1-(2-Hydroxymethylbenzyl)-N-methyl-1,2,3,4-tetrahydroisoquinolines, e. g. 1 are converted with ethyl chloroformate (ECF) to 3-phenylisochromans, e. g. 2, (mainly) by an intramolecular $\mathrm{S}_{\mathrm{N}}$-reaction with inversion at C-1 of the tetrahydroisoquinoline moiety ${ }^{1}$. In 19732), however, we concluded that a carbenium ion might be an intermediate because optically pure 1 , when treated with ECF led to crystals of $2, \mathrm{mp} .149-150^{\circ} \mathrm{C}$ (authentic 2-racemate: $\mathrm{mp} .151^{\circ} \mathrm{C}^{2)}$ ) showing an IR-spectrum (KBr) superimposible with that of racemic 2. Even in high concentrations these crystals did not show any optical activity in their CD-spectrum, and their ORD-spectrum revealed only a slight deviation from the baseline. - Later we found that the crude product 2 shows optical activity, and the optical purity was determined by an isotope dilution method using ${ }^{3} \mathrm{H}-2$ to be $82 \%{ }^{3}$. In addition, the crystals mentioned above do rotate plane-polarized light when high concentrations in another solvent $\left(\mathrm{CHCl}_{3}\right)$ and an increased length of light path through the solution were applied for polarimetric measurement ${ }^{3}$.

These results need an explanation. We repeated the conversion of (+)-1 to (+)-2, separated the crystals, did not recrystallize them (m. p. $144^{\circ} \mathrm{C} ;[\alpha]^{20}=+21^{\circ}$ ) and determined their optical purity by mass spectrometric isotope dilution analysis (MS-IDA), using Gerlach's idea who determined the optical purity of benzylamines ${ }^{4}$.

The preparation of the compounds used in these experiments is shown and explained in Scheme 1.

In quantitative ms-analysis the overall error for the determination results from the cumulative effect of the errors introduced by different parts of the system ${ }^{6}$. Therefore, we constructed calibration curves covering the range of samples likely to be encountered for quantitative determination. Mixtures of unlabeled and labeled racemic isochromans 2 and $\mathrm{D}_{2}-2$ were primarily measured at 70 and $12 \mathrm{eV}$, respectively. To this end 2 and $D_{2}-2$ were mixed in nine different molar ratios (table 1), each mixture was recrystallized four times

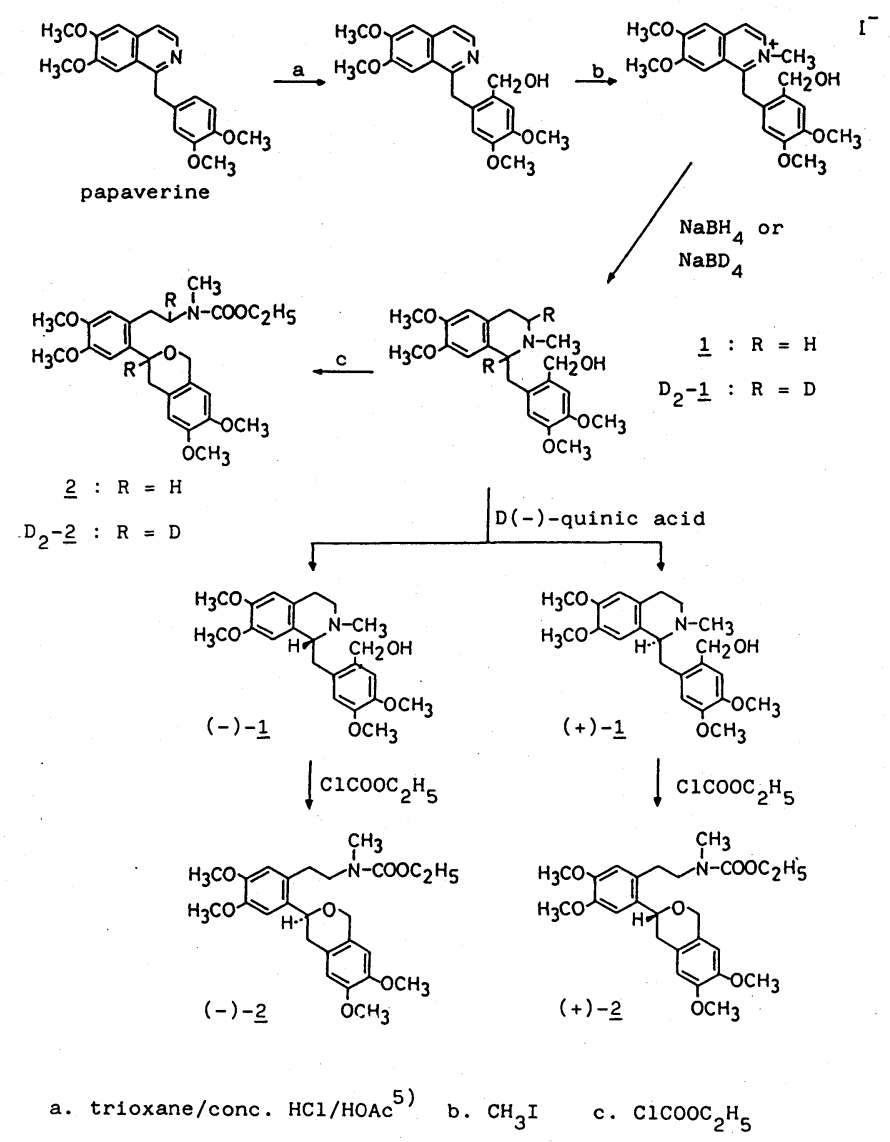

from isopropanol and measured at least $30 \times$ at 70 and $12 \mathrm{eV}$. Peak intensities of the averages were calculated and corrected for the ${ }^{13} \mathrm{C}$ satellite, in order to avoid difficulties

Tab. 1: Mixtures of ( \pm )-2 and ( \pm )- $\mathrm{D}_{2}-2$

\begin{tabular}{lccccc}
\hline Sample & $( \pm)-2$ & $( \pm)-\mathrm{D}_{2}-2$ & Sample & $( \pm)-2$ & $( \pm)-\mathrm{D}_{2}-2$ \\
\hline 1 & $0.0501 \mathrm{mmol}$ & $0.0502 \mathrm{mmol}$ & 6 & $0.0406 \mathrm{mmol}$ & $0.0605 \mathrm{mmol}$ \\
2 & $0.0605 \mathrm{mmol}$ & $0.0413 \mathrm{mmol}$ & 7 & $0.0304 \mathrm{mmol}$ & $0.0704 \mathrm{mmol}$ \\
3 & $0.0706 \mathrm{mmol}$ & $0.0307 \mathrm{mmol}$ & 8 & $0.0200 \mathrm{mmol}$ & $0.0806 \mathrm{mmol}$ \\
4 & $0.0805 \mathrm{mmol}$ & $0.0207 \mathrm{mmol}$ & 9 & $0.0103 \mathrm{mmol}$ & $0.0900 \mathrm{mmol}$ \\
5 & $0.0901 \mathrm{mmol}$ & $0.0101 \mathrm{mmol}$ & & & \\
\hline
\end{tabular}

\footnotetext{
**) Herrn Prof. Dr. Dr. h. c. mult. H. Böhme zum 80. Geburtstag in Verehrung gewidmet.
} 
which may arise with multiply labeled compounds $\left({ }^{2} \mathrm{H}\right.$ plus ${ }^{13} \mathrm{C}$; average abundance 0.015 and $1.11 \%$ for ${ }^{2} \mathrm{H}$ and ${ }^{13} \mathrm{C}$, respectively). Therefore, the contribution of ${ }^{13} \mathrm{C}$ containing ions to $\mathrm{m} / \mathrm{z} 459\left(\mathrm{M}^{+\cdot}\right.$ of 2$)$ has been substracted according to $S e i b l^{7)}$ :

$$
\begin{aligned}
& I(M+2)=\frac{(1.1 \cdot n)^{2}}{200}=3.8 \% I M \\
& I M=\text { Intensity of } \mathrm{m} / \mathrm{z} 459(2) \\
& n=25(2 \text { contains } 25 \text { C-atoms })
\end{aligned}
$$

In sample 1 (table 1), for instance, $\mathrm{m} / \mathrm{z} 459$ and $\mathrm{m} / \mathrm{z} 461$ show $161.0 \mathrm{~mm}$ and $165.9 \mathrm{~mm}$ as average peak height. Thus, the corrected intensity for $\mathrm{m} / \mathrm{z} 461$ corresponds to

$[165.9-(161.0 \cdot 0.038)]=159.8 \mathrm{~mm}$, and therefore, the isotope ratio to

$$
\text { I } \frac{\mathrm{d}_{2}}{\mathrm{~d}_{\mathrm{o}}}=\frac{159.8}{161.0}=0.993(\text { Table } 2)
$$

The values of Table 2 afford the calibration curves (Fig. 1 ), which prove that $D_{2}-2$ can be used as an internal standard $^{6}$.

Tab. 2: Calculated data of molar ratio and isotope ratio for mixtures of labeled and unlabeled standards (2) 1-9 (70/12 eV)

\begin{tabular}{cccc}
\hline Sample & Mol $\frac{\mathrm{d}_{2}}{\mathrm{~d}_{\mathrm{o}}}$ & $\mathrm{I} \frac{\mathrm{d}_{2}}{\mathrm{~d}_{\mathrm{o}}}(70 \mathrm{eV})$ & $\mathrm{I} \frac{\mathrm{d}_{2}}{\mathrm{~d}_{\mathrm{o}}}(12 \mathrm{eV})$ \\
\hline 1 & 1.002 & 1.038 & 0.993 \\
2 & 0.683 & 0.725 & 0.687 \\
3 & 0.435 & 0.483 & 0.445 \\
4 & 0.257 & 0.312 & 0.275 \\
5 & 0.112 & 0.163 & 0.127 \\
6 & 1.490 & 1.517 & 1.471 \\
7 & 2.316 & 2.297 & 2.246 \\
8 & 4.030 & 3.889 & 3.841 \\
9 & 8.738 & 8.143 & 8.375 \\
\hline
\end{tabular}

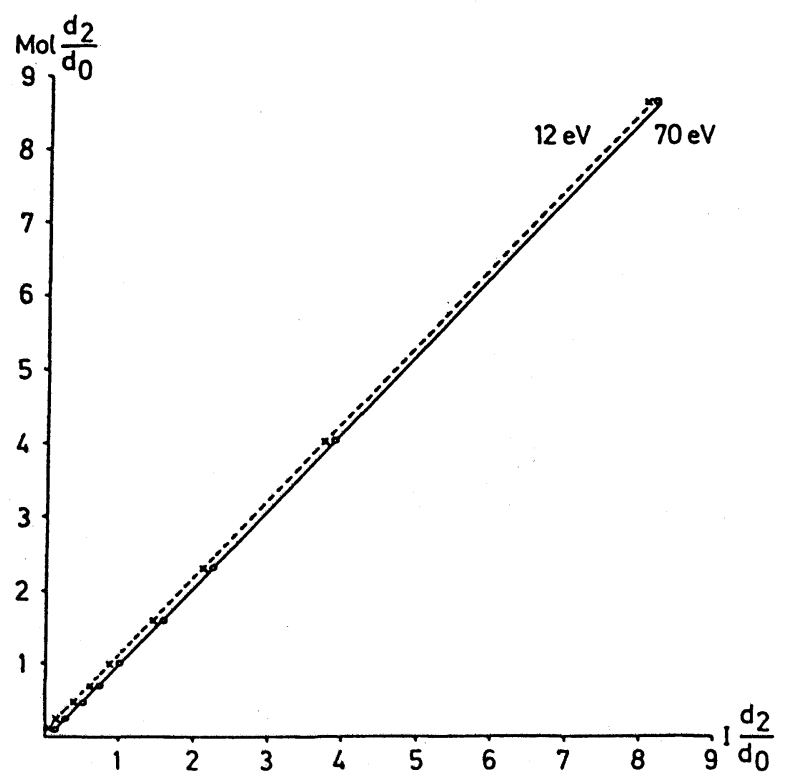

Fig. 1: Calibration curves
To determine the optical purity of the crystals of (+)-2 $\left([\alpha]^{28} \mathrm{~B}=+21^{\circ}\right) 0.0506 \mathrm{mmol}$ of $(+)-2$ and $0.0505 \mathrm{mmol}$ of (racemic) labeled isomer $\mathrm{D}_{2}-2$ were mixed and recrystallized four times from isopropanol until no more optical activity is shown. This mixture of racemates was measured as indicated for the samples 1-9 to obtain the values $1.014(70 \mathrm{eV})$ and $1.015(12 \mathrm{eV})$ for the isotope ratio $I \mathrm{~d}_{2} / \mathrm{d}_{0}$. The optical purity was calculated using Berson's equation ${ }^{8)}$ which had also been used in lit. ${ }^{3)}$.

$E=\left[(X+B)^{2}-\left(C_{0} / C\right) \cdot\left(X^{2}+B X\right)\right]^{1 / 2}$

$\%$ optical purity $=\mathrm{E} \cdot 100 / \mathrm{B}$

B: weight of the test sample ((+)-2)

E: excess of one enantiomer in B

$X$ : weight of the labeled racemate added to the test sample $\left(D_{2}-2\right)$

$\mathrm{C}_{\mathrm{o}}$ : specific activity*) of the labeled racemate added to the test sample

C: specific activity ${ }^{*}$ of the reisolated racemate.

$B=23.26 \mathrm{mg} ; X=23.33 \mathrm{mg} ; C_{o}=100 \% \mathrm{~d}_{2}$;

$\mathrm{C}=\mathrm{I} \mathrm{d}_{2} / \mathrm{d}_{\mathrm{o}}=1.015$ (or 1.014$)=50.4 \% \mathrm{~d}_{2}$,

therefore, $E=4.30$ and the optical purity $=18.5 \%$.

Obviously in this case the racemate (racemic mixture) of $\mathbf{2}$ is enriched by crystallization.

*) Instead of the specific (radio)activity we used the D-content.

\section{Experimental Part}

Mp.: uncorrected, Büchi SMP-20. - Elementary analysis: Microanalytical Laboratory of the Univ. of Regensburg. $-I R(\mathrm{KBr})$ : Beckman Acculab III. $-{ }^{1} \mathrm{H}$-NMR: Varian EM $390(90 \mathrm{MHz}), 30^{\circ} \mathrm{C}$, TMS int. stand. UV: Uvikon 810, MeOH (Uvasol „Merck“). - MS: Varian MAT CH5 and $311 \mathrm{~A}$.

\section{6'-Hydroxymethylpapaverine: lit.5) \\ $6^{\prime}$-Hydroxymethylpapaverine- $N$-methyliodide: lit. ${ }^{2)}$ \\ $6^{\prime}$-Hydroxymethyllaudanosine (1)}

$15.3 \mathrm{~g}(0.03 \mathrm{~mol}) 6^{\prime}$-hydroxymethylpapaverine- $\mathrm{N}$-methyliodide dissolved in $900 \mathrm{ml} 70 \% \mathrm{EtOH}$ were added dropwise to a stirred suspension of $5.0 \mathrm{~g}$ $(0.12 \mathrm{~mol}) \mathrm{NaBH}_{4}$ in $90 \mathrm{ml} 70 \% \mathrm{EtOH}$ at $0{ }^{\circ} \mathrm{C}$. After refluxing for $4 \mathrm{~h}$, the org. layer was evaporated and the remaining aqueous layer was extracted with $\mathrm{CHCl}_{3}$. Drying and removal of the solvent led to an oily product, colourless needles from $\mathrm{Et}_{2} \mathrm{O}: 6.4 \mathrm{~g}(55 \%), \mathrm{mp} .99-100^{\circ} \mathrm{C}$ $\left(103-104{ }^{\circ} \mathrm{C}^{2}\right)$ ). $-{ }^{1} \mathrm{H}-\mathrm{NMR}: \delta(\mathrm{ppm})=2.17-3.23\left(\mathrm{~m} ; 7 \mathrm{H},-\mathrm{CH}_{2}-\right.$ and $\mathrm{H}-$ 1), $2.3\left(\mathrm{~s} ; 3 \mathrm{H},-\mathrm{NCH}_{3}\right), 3.63\left(\mathrm{~s} ; 3 \mathrm{H},-\mathrm{OCH}_{3}\right), 3.83\left(\mathrm{~s} ; 6 \mathrm{H},-\mathrm{OCH}_{3}\right), 3.88(\mathrm{~s}$; $\left.3 \mathrm{H},-\mathrm{OCH}_{3}\right), 4.43\left(\mathrm{~s} ; 2 \mathrm{H},-\mathrm{CH}_{2} \mathrm{OH}\right), 6.27,6.50,6.70$ and $6.83(4 \times \mathrm{s} ; 4 \mathrm{H}$, aromatic).

( \pm )-3-I2'-( $\beta$-N-Ethoxycarbonyl-N-methyl-aminoethyl)-4', $5^{\prime}$-dimethoxyphenyll-6,7-dimethoxyisochroman $(( \pm)-2)$.

$( \pm)-1$ was reacted with ECF as reported ${ }^{2)}$ to give ( \pm )-2, $\mathrm{mp} .147-148^{\circ} \mathrm{C}$ $\left(150-151^{\circ} \mathrm{C}^{2}\right)$ ). - IR: $1700 \mathrm{~cm}^{-1}(\mathrm{CO}) .-\mathrm{UV}: \lambda \max (\log \varepsilon)=282(3.99)$, 231 (4.32), $208 \mathrm{~nm}$ (4.75). $-{ }^{1} \mathrm{H}-\mathrm{NMR}: \delta(\mathrm{ppm})=1.17(\mathrm{t} ; \mathrm{J}=6 \mathrm{~Hz}, 3 \mathrm{H}$, $\left.-\mathrm{CH}_{2}-\mathrm{CH}_{3}\right), 2.73-3.63\left(\mathrm{~m} ; 7 \mathrm{H},-\mathrm{CH}_{2}-\right.$ and $\left.\mathrm{H}-3\right), 3.87\left(\mathrm{~s} ; 12 \mathrm{H},-\mathrm{OCH}_{3}\right)$, $4.07\left(\mathrm{q} ; \mathrm{J}=6 \mathrm{~Hz}, 2 \mathrm{H},-\mathrm{CH}_{2}-\mathrm{CH}_{3}\right), 4.90\left(\mathrm{~s} ; 2 \mathrm{H},-\mathrm{O}-\mathrm{CH}_{2}-\right), 6.53,6.63,6.67$ and 7.07 (4 $\times \mathrm{s} ; 4 \mathrm{H}$, aromatic).

Enantiomers of $\mathbf{1}$

$( \pm)-1$ was resolved with $\mathrm{D}-(-)$-quinic acid $\left.^{2}\right)$ to give its enantiomers.

(+)-1: mp. $125^{\circ} \mathrm{C}\left(125^{\circ} \mathrm{C}^{2)}\right),[\alpha]_{\mathrm{D}}^{20}=+88^{\circ}\left(\mathrm{c}=3.0, \mathrm{CHCl}_{3}\right)$.

(-)-1: mp. $126^{\circ} \mathrm{C}\left(124^{\circ} \mathrm{C}^{2)}\right),[\alpha]^{20} \mathrm{D}=-88^{\circ}\left(\mathrm{c}=3.0, \mathrm{CHCl}_{3}\right)$. 


\section{Enantiomers of $\mathbf{2}$}

The enantiomers of 2 were prepared from (+)-1 and (-)-1 with ECF as reported $^{1,2)}$.

( \pm )-1,3-Dideutero-6'-hydroxymethyllaudanosine $\left(( \pm)-\mathrm{D}_{2}-1\right)$

$( \pm)-D_{2}-1$ was prepared from 6 '-hydroxymethylpapaverine-N-methyliodide with $\mathrm{NaBD}_{4}$ as described for $1 ; \mathrm{mp} .99^{\circ} \mathrm{C}$.

$\mathrm{C}_{22} \mathrm{H}_{27} \mathrm{D}_{2} \mathrm{NO}_{5}(389.5)$ calcd. C $67.8 \mathrm{H} 7.03$ found C $67.8 \mathrm{H}$ 7.00. - IR: $3150 \mathrm{~cm}^{-1}(\mathrm{OH})$. - UV: $\lambda \max (\log \varepsilon)=283(3.84), 212 \mathrm{~nm}(4.42) .-{ }^{1} \mathrm{H}-$ NMR: $\delta(\mathrm{ppm})=2.30\left(\mathrm{~s} ; 3 \mathrm{H},-\mathrm{NCH}_{3}\right), 2.13-3.30(\mathrm{~m} ; 5 \mathrm{H}), 3.673 .83$, 3.85 and $3.90\left(4 \times \mathrm{s} ; 12 \mathrm{H},-\mathrm{OCH}_{3}\right), 4.43\left(\mathrm{~s} ; 2 \mathrm{H},-\mathrm{CH}_{2} \mathrm{OH}\right), 6.27,6.50$, 6.67 and $6.80(4 \times \mathrm{s} ; 4 \mathrm{H}$, aromatic). $-\mathrm{MS}(70 \mathrm{eV}): \mathrm{m} / \mathrm{z}=209(15 \%), 208$ (100), $193(5), 192(9)$.

( \pm$)-3$-Deutero-3-12'-( $\beta$-deutero- $\beta$ - $N$-ethoxycarbonyl- $N$-methylaminoethyl)-4',5'-dimethoxyphenyl) I-6,7-dimethoxyisochroman $\left(( \pm)-\mathrm{D}_{2}-2\right)$.

$( \pm)-D_{2}-2$ was prepared from $( \pm)-D_{2}-1$ with ECF as reported for undeuterated $( \pm)-2^{2)} ; \mathrm{mp} .145^{\circ}-146^{\circ} \mathrm{C}$.

$\mathrm{C}_{25} \mathrm{H}_{31} \mathrm{D}_{2} \mathrm{NO}_{7}(461.5)$ calcd. $\mathrm{C} 65.0 \mathrm{H} 6.78$ found $\mathrm{C} 65.1 \mathrm{H}$ 6.78. - IR: $1710 \mathrm{~cm}^{-1}(\mathrm{CO}) .-\mathrm{UV}: \lambda \max (\log \varepsilon)=282(3.92), 230(4.28), 212 \mathrm{~nm}$ (4.40). $-{ }^{1} \mathrm{H}-\mathrm{NMR}: \delta(\mathrm{ppm})=1.50\left(\mathrm{~s}\right.$; broad, $\left.3 \mathrm{H},-\mathrm{CH}_{2}-\mathrm{C}_{3}\right), 2.63-3.87$ (m; 5H, $-\mathrm{CH}_{2}$ - and -CH-D-), $3.87\left(\mathrm{~s} ; 12 \mathrm{H},-\mathrm{OCH}_{3}\right), 4.03(\mathrm{q} ; \mathrm{J}=6 \mathrm{~Hz}$, $\left.2 \mathrm{H},-\mathrm{CH}_{2}-\mathrm{CH}_{3}\right), 6.57,6.63,6.67$ and $7.07(4 \times \mathrm{s} ; 4 \mathrm{H}$, aromatic). - MS $(70 \mathrm{eV}): \mathrm{m} / \mathrm{z}=461\left(\mathrm{M}^{+\cdot}, 7 \%\right), 443(1, * 425.70), 358(5), 340(5), 180(5)$, 165 (14), 164 (100), $149\left(7,{ }^{*} 135.37\right), 117$ (7).

\section{Literature}

1 W. Wiegrebe, S. Prior, and K. K. Mayer, Arch. Pharm. (Weinheim) 315, 262 (1982).

2 W. Wiegrebe, H. Reinhart, and J. Fricke, Pharm. Acta Helv. 48, 420 (1973).

3 H. M. Stephan, G. Langer, and W. Wiegrebe, Pharm. Acta Helv. 51, 164 (1976).

4 H. Gerlach, Helv. Chim. Acta 49, 2481 (1966).

5 P. Mathieu, J. Gardent, and M. M. Janot, C. R. Acad. Sci. Ser. C. 267, 1416 (1968), we use trioxane instead of $\mathrm{H}_{2} \mathrm{CO}$; cf. S. Prior, W. Wiegrebe, and G. Sariyar, Arch. Pharm. (Weinheim) 315, 280 (1982).

6 B. J. Millard, Quantitative Mass Spectrometry. Chapters 3 and 4, Heyden \& Son. Ltd., London 1978.

7 J. Seibl, Massenspektrometrie, p. 21, Akademische Verlagsges., Frankfurt/M. 1970.

8 J.A. Berson and D. A. Ben-Efraim, J. Am. Chem. Soc. 81, 4083(1959). 